(NASA-CR-200043) AN IMPROVED

N96-17865

QUASISTATIC LINE-SHAPE THEORY: THE

EFFECTS OF MOLECULAR MOTION ON THE

LINE WINGS (NASA. Goddard Inst.

for space Studies) $13 \mathrm{p}$

Unclas

$63 / 25 \quad 0098271$ 


\title{
An improved quasistatic line-shape theory: The effects of molecular motion on the line wings
}

\author{
Q. Ma \\ Department of Applied Physics, Columbia University and Institute for Space Studies, Goddard Space Flight \\ Center, New York, New York 10025 \\ R. H. Tipping \\ Department of Physics and Astronomy, University of Alabama, Tuscaloosa, Alabama 35487
}

(Received 25 October 1993; accepted 4 January 1994)

A theory is presented for the modification of the line-shape functions and absorption coefficient due to the breakdown of the quasistatic approximation. This breakdown arises from the effects of molecular motion and increases the absorption in the near wings. Numerical calculations for the high-frequency wing of the $\nu_{3}$ band of $\mathrm{CO}_{2}$ broadened by Ar are reported and it is shown that these effects are significant near the bandhead. The importance of such corrections in other spectral regions and for other systems is discussed briefly.

\section{INTRODUCTION}

The calculation of accurate frequency profiles for molecular vibration-rotational transitions valid over a range of different physical parameters (e.g., number density, temperature, etc.) remains an unsolved problem. Various theories valid for small frequency displacements from the resonant line center have been proposed over the years. ${ }^{1}$ For instance, the impact theory in which the duration of collisions is assumed to be zero leads to Lorentzian-shaped profiles that apply quite generally to non-overlapping lines arising in low density gases. ${ }^{2.3}$ On the other hand, only relatively recently have tractable theories been proposed that are valid for large frequency displacements. Rosenkranz, ${ }^{4,5}$ starting the basic formalism given by Fano, ${ }^{6}$ has developed a far-wing theory based on the quasistatic and binary collision approximations and was able to obtain theoretical results for self- and $\mathrm{N}_{2}$-broadened $\mathrm{H}_{2} \mathrm{O}$ spectra (the so-called continuum absorption) which are in substantial agreement with the experimental data. ${ }^{7}$ But because of the assumptions made, this theory is applicable only to the high-frequency wing of the pure rotational band. Rosenkranz's theory has been subsequently extended and improved by other authors. ${ }^{8-13}$ Boulet et al. ${ }^{8}$ proposed a line-by-line generalization of Rosenkranz's theory, but because of the extensive computations involved this approach has been implemented only for atom-molecule systems. ${ }^{14} \mathrm{Ma}$ and Tipping ${ }^{9-13}$ in a series of papers have considered a number of improvements, removed the restriction for the applicability only to high-frequency wings, and applied their results to the calculation of self- and foreignbroadened $\mathrm{H}_{2} \mathrm{O}$ for frequencies up to $10000 \mathrm{~cm}^{-1}$. Their results are generally in good agreement with both laboratory and atmospheric data. ${ }^{9-13,15}$ However, in all the formalisms mentioned above, the time dependence of the interaction between an absorber and a perturber is ignored. .

In the present paper we consider corrections to the quasistatic theory implicit in all the theoretical treatments discussed above. ${ }^{+.5 .8-15}$ In Sec. II, we present the general formalism and give results for the effects of molecular motion (neglected in the quasistatic approximation) on the spectral density and on the line shapes. This formalism is applied in
Sec. III to the case of the $\nu_{3}$ band of $\mathrm{CO}_{2}$ broadened by Ar. A discussion of the numerical results and conclusions of the present work are presented on Sec. IV.

\section{THEORY}

\section{A. The basic approximations}

In order to take into account the molecular motion for the wing region, we make some basic approximations besides the binary collision approximation.

(1) The quasistatic approximation. In the quasistatic approximation, the duration of a collision is assumed to be infinite and the positions of interacting molecules are fixed in space during the period of time of interest. One usually treats the relative motion of the two molecules classically; i.e., the ensemble average over the relative motion is carried out by integrating over $r$, the separation between two interacting molecules, with a statistical weight for a pair of molecules $\rho^{\text {(int) }}(\mathbf{r})$ related to the interaction between them. We note that since the interaction is independent of time, the molecular motion is totally ignored. This approximation limits the applicability of the theory to the far-wing regions. In the nearwing region, although we can still assume the duration of the collision is very large, we cannot take it as infinite as we did previously. Therefore, the molecular motion should be taken into account during the time of interest.

We assume the duration of collision, $\tau$, is very large. Therefore, during the time of interest $(t \sim 1 / \Delta \omega$, which is small), the displacements of molecules are small and the interaction between them changes only slightly. We are thus able to write the interaction as two components: a main component $V$ independent of time and a small correction term $V_{1}(t)$ that is dependent on time. Consequently, the total Hamiltonian of two interacting molecules similarly consists of two parts, a main part independent of time and a small time-dependent correction term

$$
H(t)=H_{a}+H_{b}+V+V_{1}(t) \equiv H+V_{1}(t) ;
$$

here the subscripts $a$ and $b$ refer to the absorber and perturber, respectively. 
(2) The closest region approximation. The interaction between two molecules depends on their separation. In general, during the whole collision process, the closer the distance between two interacting molecules becomes, the stronger the anisotropic interaction. As is well known, the anisotropic interaction is mainly responsible for collision broadening which is related to the coupling between the interaction and the transition of the absorber molecule during the collision process. Therefore, the strongest coupling effect takes place when two interacting molecules are near the separation of closest approach (we choose that time as $t=0$ ). In comparison with other parts of the collision trajectory, the contribution of this coupling comes mainly from the closest region, and we can assume that it can be ignored elsewhere.

(3) The classical ensemble average approximation. We do not treat the relative translational motion of two interacting molecules quantum mechanically, rather we consider their motion as a classical trajectory. In this case, the ensemble average over the relative motion is an ensemble average over all classical trajectories. However, based on the closest region approximation, the coupling effects from the whole trajectory arise mainly from separations around the closest point $\mathbf{r}_{0}$. We introduce a parameter $\eta$ to estimate the fraction of the trajectory consistent with the closest region approximation that contributes significantly. With a straight line trajectory approximation, one is able to carry out this ensemble average. Based on the approximations discussed above, we can develop a formalism to take into account the effects of the molecular motion on the line wing shapes.

\section{B. General formalism} by

The correlation function of the dipole moment is defined

$$
\left\langle\boldsymbol{\mu}_{a}^{(H)}(0) \cdot \boldsymbol{\mu}_{a}^{(H)}(t)\right\rangle=\sum_{i} \rho_{i}\left\langle i\left|\boldsymbol{\mu}_{a}^{(H)}(0) \cdot \boldsymbol{\mu}_{a}^{(H)}(t)\right| i\right\rangle,
$$

where $\rho_{i}$ is the normalized Boltzmann factor of the initial state $i$. In the above expression, $\mu_{a}^{(H)}(t)$ is the dipole operator of the absorber molecule in the Heisenberg representation which satisfies the equation

$$
\frac{d}{d t} \mu_{a}^{(H)}(t)=i\left[H(t), \mu_{a}^{(H)}(t)\right] / \hbar,
$$

where the square brackets indicate commutating operator. In the present case, the Hamiltonian $H(t)$ explicitly depends on time and we are unable to simply express $\mu_{a}^{(H)}(t)$ as $e^{i H(t) t / \hbar} \mu_{a}^{(H)}(0) e^{-i H(t) t / \hbar}$ as we did previously. However, we are able to solve Eq. (3) using a perturbational method as follows. For simplicity, we will omit the subscript $a$ of $\mu_{a}^{(H)}(t)$ indicating the absorber molecule and the superscript $(H)$ indicating the Heisenberg representation. We introduce a time displacement operator $U(t)$ and assume that

$$
\boldsymbol{\mu}(t)=U(t) \boldsymbol{\mu}(0) U(t)^{\dagger}
$$

With Eqs. (3) and (4), we obtain

$$
\frac{d}{d t} U(t)=i H(t) U(t) / \hbar \text {. }
$$

From Eq. (1) and the perturbational method, we obtain

$$
\begin{aligned}
U(t)= & e^{i H t / \hbar}\left\{1+i \int_{0}^{t} V_{1}^{\prime}\left(t^{\prime}\right) d t^{\prime}\right. \\
& \left.+(i)^{2} \int_{0}^{t} V_{1}^{\prime}\left(t^{\prime}\right) d t^{\prime} \int_{0}^{t^{\prime}} V_{1}^{\prime}\left(t^{\prime \prime}\right) d t^{\prime \prime}+\cdots\right\},
\end{aligned}
$$

where

$$
V_{1}^{\prime}(t) \equiv e^{-i H t / \hbar} V_{1}(t) e^{i H t / \hbar}=e^{-i L t} V_{1}(t) .
$$

In the above expression, we have introduced the Liouville operator $L$ defined by

$$
L=\left(H I^{*}-I H^{*}\right) / \hbar \text {. }
$$

Then, we have

$$
\begin{aligned}
\mu(t)= & e^{i H t}\left\{1+i \int_{0}^{t} V_{1}^{\prime}\left(t^{\prime}\right) d t^{\prime}+\cdots\right\} \mu(0) \\
& \times\left\{1-i \int_{0}^{t} V_{1}^{d}\left(t^{\prime}\right) d t^{\prime}+\cdots\right\} e^{-i H t} \\
= & e^{i L t} \mu(0)+i e^{i H t}\left[\int_{0}^{t} V_{1}^{l}\left(t^{\prime}\right) d t^{\prime}, \mu(0)\right] e^{-i H t}+\cdots \\
= & \mu_{0}(t)+i e^{i L t}\left[\int_{0}^{t} V_{1}^{\prime}\left(t^{\prime}\right) d t^{\prime}, \mu(0)\right]+\cdots,
\end{aligned}
$$

where

$$
\mu_{0}(t) \equiv e^{i H t} \mu(0) e^{-i H t}=e^{i L t} \mu(0) .
$$

It is obvious that

$$
\mu_{0}(0)=\mu(0) \text {. }
$$

The correlation function can be written as

$$
\begin{aligned}
\operatorname{Tr}\{\rho \mu(0) \cdot \mu(t)\}= & \operatorname{Tr}\left\{\rho \mu_{0}(0) \cdot e^{i L t} \mu_{0}(0)\right\}+\operatorname{Tr}\left\{\rho \mu_{0}(0)\right. \\
& \left.\cdot i e^{i L t}\left[\int_{0}^{t} V_{1}^{\prime}\left(t^{\prime}\right) d t^{\prime}, \mu_{0}(0)\right]\right\} \\
& +\cdots,
\end{aligned}
$$

and its Fourier transform, the spectral density $F(\omega)$, as

$$
\begin{aligned}
F(\omega)= & \frac{1}{2 \pi} \int_{-\infty}^{\infty} e^{i \omega t} \operatorname{Tr}\{\rho \mu(0) \cdot \mu(t)\} d t=F_{0}(\omega) \\
& +F_{1}(\omega)+\cdots .
\end{aligned}
$$

The zero-order term of the spectral density, $F_{0}(\omega)$, is defined by

$$
\begin{aligned}
F_{0}(\omega) & =\frac{1}{2 \pi} \int_{-\infty}^{\infty} e^{i \omega t} \operatorname{Tr}\left\{\rho \mu_{0}(0) \cdot e^{i L t} \mu_{0}(0)\right\} d t \\
& =\frac{1}{\pi} \operatorname{Re} \int_{0}^{\infty} e^{i \omega t} \operatorname{Tr}\left\{\mu \cdot e^{-i L t} \rho \mu\right\} d t,
\end{aligned}
$$


where for simplifying the notation, we have omitted the subscript and the zero-time argument of the dipole moment operator $\mu_{0}(0)$. The first-order term of the spectral density can be expressed as (see Appendix A)

$$
\begin{aligned}
F_{1}(\omega) & =\frac{1}{2 \pi} \int_{-\infty}^{\infty} e^{i \omega t} \operatorname{Tr}\left\{\rho \mu \cdot i e^{i L t}\left[\int_{0}^{t} V_{1}^{\prime}\left(t^{\prime}\right) d t^{\prime}, \mu\right]\right\} d t, \\
& =\frac{1}{\pi} \operatorname{Re} \int_{0}^{\infty} e^{i \omega t} \operatorname{Tr}\left\{\mu \cdot e^{-i L t} \bar{V}_{1}(t) \frac{1}{\omega-L} \rho \mu\right\} d t,
\end{aligned}
$$

where $\bar{V}_{1}(t)$ is a Liouville operator corresponding to $V_{1}(t)$.

It is well known $n^{9-13}$ that since the isotropic interaction does not depend on the rotational quantum numbers, the Liouville operator associated with it will cancel out when it acts on the line space. Therefore, the isotropic interaction need not be included in the Liouville operator $L$ nor in its time-dependent correction part $\bar{V}_{1}(t)$.

\section{The interaction at the closest reglon}

In the present paper, we assume that the anisotropic interaction takes a product form

$$
V_{\text {aniso }}[r(t)] \equiv R[r(t)] G_{\text {aniso }}[\theta(t), \varphi(t)]
$$

where $r(t), \theta(t)$, and $\varphi(t)$ are the spherical coordinate components of $r(t)$. We note that for simplifying the notation, the orientations of the two interacting molecules have not been explicitly indicated in the above expression. Since the vector $r(t)$ is time dependent, the anisotropic interaction explicitly depends on time. However, based on the closest region and the quasistatic approximations, we are only interest in the behavior of this interaction around the closest point $r_{0}$ and for time $t \ll \tau$. We choose $r_{0}$ as the $k$ axis of a space-fixed frame $(i, j$, and $\mathbf{k})$ and $r(t)$ is on the plane with $\varphi=0$. We can expand $V_{\text {aniso }}(r, \theta)$ around $t=0$ as

$$
\begin{aligned}
V_{\text {aniso }}(r, \theta)= & V_{\text {aniso }}\left(r_{0}, 0\right)+\left.t\left(\frac{d r}{d t} \frac{\partial}{\partial r}+\frac{d \theta}{d t} \frac{\partial}{\partial \theta}\right) V_{\text {aniso }}(r, \theta)\right|_{t=0}+\frac{1}{2} t^{2}\left[\left(\frac{d r}{d t}\right)^{2} \frac{\partial^{2}}{\partial r^{2}}+2\left(\frac{d r}{d t}\right)\left(\frac{d \theta}{d t}\right) \frac{\partial^{2}}{\partial r \partial \theta}+\frac{d^{2} r}{d t^{2}} \frac{\partial}{\partial r}\right. \\
& \left.+\left(\frac{d \theta}{d t}\right)^{2} \frac{\partial^{2}}{\partial \theta^{2}}+\frac{d^{2} \theta}{d t^{2}} \frac{\partial}{\partial \theta}\right]\left.V_{\text {aniso }}(r, \theta)\right|_{t=0}+\cdots \\
= & V_{\text {aniso }}\left(r_{0}, 0\right)+t \frac{d \theta}{d t} \frac{\partial}{\partial \theta} V_{\text {aniso }}\left(r_{0}, 0\right) \\
& +\frac{1}{2} t^{2}\left[\frac{d^{2} r}{d t^{2}} \frac{\partial}{\partial r}+\left(\frac{d \theta}{d t}\right)^{2} \frac{\partial^{2}}{\partial \theta^{2}}+\frac{d^{2} \theta}{d t^{2}} \frac{\partial}{\partial \theta}\right] V_{\text {aniso }}\left(r_{0}, 0\right)+\cdots,
\end{aligned}
$$

where in the last step of deriving Eq. (17) we have used the fact that $(d r / d t)=0$ at the closest point. Since the set of trajectories has rotational symmetry with respect to the axis $\mathbf{k}$, we expect that the contribution from the second term will be zero and we will simply omit this term in Eq. (17). Consequently, we are able to express $V_{\text {aniso }}(r, \theta)$ as two parts: a dominant part $V_{\text {aniso }}\left(r_{0}, 0\right)$ which is independent of the time and a small correction part which is proportional to $t^{2}$. It is worth mentioning that the correction term is an even function of time. Thus, we rewrite $V_{1}(t)$ as

$$
V_{1}(t)=t^{2} V_{1}^{\prime}
$$

where

$$
\begin{aligned}
V_{1}^{\prime}= & \frac{1}{2}\left[\frac{d^{2} r}{d t^{2}} \frac{\partial}{\partial r} V_{\text {aniso }}\left(r_{0}, 0\right)+\left(\frac{d \theta}{d t}\right)^{2} \frac{\partial^{2}}{\partial \theta^{2}} V_{\text {aniso }}\left(r_{0}, 0\right)\right. \\
& \left.+\frac{d^{2} \theta}{d t^{2}} \frac{\partial}{\partial \theta} V_{\text {aniso }}\left(r_{0}, 0\right)\right] .
\end{aligned}
$$

Based on classical dynamics, we have

$m \frac{d^{2} r}{d t^{2}}=m r\left(\frac{d \theta}{d t}\right)^{2}-\frac{d}{d r} V_{\text {iso }}(r)-\frac{\partial}{\partial r} V_{\text {aniso }}(r, \theta)$, $m\left(2 \frac{d r}{d t} \frac{d \theta}{d t}+r \frac{d^{2} \theta}{d t^{2}}\right)=-\frac{1}{r} \frac{\partial}{\partial \theta} V_{\text {aniso }}(r, \theta)$,

where $\boldsymbol{m}$ is the reduced mass of the two interacting molecules. Then, we obtain

$$
\begin{aligned}
\left.\frac{d^{2} r}{d t^{2}}\right|_{r=r_{0}, \theta=0}= & r_{0}\left(\frac{d \theta}{d t}\right)^{2}-\frac{1}{m} \frac{d}{d r} V_{\text {iso }}\left(r_{0}\right)-\frac{1}{m} \frac{\partial}{\partial r} V_{\text {aniso }}\left(r_{0}, 0\right) \\
= & \frac{1}{r_{0}} v_{\infty}^{2}-\frac{2}{m r_{0}} V_{\text {iso }}\left(r_{0}\right)-\frac{2}{m r_{0}} V_{\text {aniso }}\left(r_{0}, 0\right) \\
& -\frac{1}{m} \frac{d}{d r} V_{\text {iso }}\left(r_{0}\right)-\frac{1}{m} \frac{\partial}{\partial r} V_{\text {aniso }}\left(r_{0}, 0\right)
\end{aligned}
$$

In the last step of deriving Eq. (22), conservation of the energy

$$
\frac{1}{2} m r_{0}^{2}\left(\frac{d \theta}{d t}\right)^{2}=\frac{1}{2} m v_{\infty}^{2}-V_{\text {iso }}\left(r_{0}\right)-V_{\text {aniso }}\left(r_{0}, 0\right),
$$

has been taken into account. In the above expression, $v_{\infty}$ is an initial relative velocity when the separation between two interacting molecules is infinity. Since $\frac{1}{2} m r_{0}^{2}(d \theta / d t)^{2} \geqslant 0$, the range of $r_{0}$ which can be approached is limited. This fact will be used later. From Eq. (21) we obtain 


$$
\left.\frac{d^{2} \theta}{d t^{2}}\right|_{r=r_{0}, \theta=0}=-\frac{1}{m r_{0}^{2}} \frac{\partial}{\partial \theta} V_{\text {aniso }}\left(r_{0}, 0\right) .
$$

Using Eqs. (22) and (24), $V_{1}^{\prime}$ can be expressed as

$$
\begin{aligned}
V_{\mathrm{i}}^{\prime}= & \frac{1}{2 m r_{0}}\left\{m v_{\infty}^{2}-\left(2+r_{0} \frac{d}{d r}\right)\left[V_{\text {iso }}\left(r_{0}\right)\right.\right. \\
& \left.\left.+V_{\text {aniso }}\left(r_{0}, 0\right)\right]\right\} \frac{\partial}{\partial r} V_{\text {aniso }}\left(r_{0}, 0\right) \\
& +\frac{1}{2}\left(\frac{d \theta}{d t}\right)^{2} O^{2} V_{\text {aniso }}-\frac{1}{2 m r_{0}^{2}}\left(O V_{\text {aniso }}\right)^{2},
\end{aligned}
$$

where $O^{2} V \equiv \partial^{2} V / \partial \theta^{2}$ and $(O V)^{2} \equiv(\partial V / \partial \theta)^{2}$. We note that the first term of Eq. (25) depends on the velocity $v_{\infty}$ while the last term is related to a torque. ${ }^{16}$ For simplifying the notation, we will omit the subscript of $r_{0}$ in the following discussion.

\section{The effect of the molecular motion on the expression of $F_{1}(\omega)$}

In a previous paper, ${ }^{10}$ we have derived an expression for $F_{0}(\omega)$ in which, at least formally, the bath variables have been isolated

$$
\begin{aligned}
F_{0}(\omega)= & \frac{1}{\pi} \ln \frac{1}{2 \pi i} \int_{-\infty}^{\infty} d \omega^{\prime} \operatorname{Tr}\left[\mu \cdot \frac{1}{\omega-\omega^{\prime}-L_{a}}\right. \\
& \left.\times\left\langle\frac{1}{\omega^{\prime}-L_{1}}\right\rangle_{b}^{\rho^{(a)} \mu}\right]_{a}
\end{aligned}
$$

where

$$
\left\langle\frac{1}{\omega^{\prime}-L_{1}}\right\rangle_{b}=\operatorname{Tr}\left[\frac{1}{\omega^{\prime}-L_{1}} \rho^{(b)}\right]_{b} .
$$

In the above expressions we explicitly denote the summation indices of the trace, $a$ or $b$, for the absorber or bath molecular variables, respectively.

Now we focus on the correction term $F_{1}(\omega)$. With Eqs. (A4), (AS) and (18), we have

$$
\begin{aligned}
F_{1}(\omega) & =\frac{1}{\pi} \operatorname{Re} \operatorname{Tr} \int_{0}^{\infty}\left[\mu \cdot t^{2} e^{i(\omega-L) t} \bar{V}_{1}^{\prime} \frac{1}{\omega-L} \rho \mu\right] d t, \\
& \simeq \frac{1}{\pi} \operatorname{Re} \operatorname{Tr} \int_{0}^{\infty}\left[\mu \cdot t^{2} e^{i\left(\omega-L_{0}\right) t} \bar{V}_{1}^{\prime} \frac{1}{\omega-L} \rho \mu\right] d t,
\end{aligned}
$$

where the time-independent Liouville operator $L$ consists of two components,

$$
L=L_{0}+L_{1} .
$$

corresponding to the unperturbed Hamiltonian of the absorber and bath molecule and the time-independent interaction, $V_{\text {aniso }}\left(r_{0}, 0\right)$, respectively. We assume that

$$
e^{-i L t} \simeq e^{-i L_{0} t} e^{-i L_{1} t},
$$

where the limitation of this approximation has been discussed in a previous paper. ${ }^{10}$ Based on it, we can rewrite the resolvent operator $[1 /(\omega-L)]$ in Eq. (28) as

$$
\begin{aligned}
\frac{1}{\omega-L} & =-i \int_{0}^{\infty} e^{-i L_{1} t} e^{i\left(\omega-L_{0}\right) t} d t \\
& =-\frac{1}{2 \pi i} \int_{-\infty}^{\infty} d \omega^{\prime} \frac{1}{\omega^{\prime}-L_{1}} \times \frac{1}{\omega-\omega^{\prime}-L_{0}} .
\end{aligned}
$$

Then $F_{1}(\omega)$ can be expressed as

$$
\begin{aligned}
F_{1}(\omega) & =-\frac{1}{\pi} \operatorname{Re} \frac{1}{2 \pi i} \int_{-\infty}^{\infty} d \omega^{\prime} \operatorname{Tr}\left[\mu \cdot \int_{0}^{\infty} t^{2} e^{i\left(\omega-L_{0}\right) t} d t \bar{V}_{1}^{\prime} \frac{1}{\omega^{\prime}-L_{1}} \times \frac{1}{\omega-\omega^{\prime}-L_{0}} \rho \mu\right] \\
& =\frac{1}{\pi} \operatorname{Re} \frac{1}{2 \pi i} \int_{-\infty}^{\infty} d \omega^{\prime} \operatorname{Tr}\left[\mu \cdot \frac{d^{2}}{d \omega^{2}} \int_{0}^{\infty} e^{i\left(\omega-L_{0}\right) t} d t \bar{V}_{1}^{\prime} \frac{1}{\omega^{\prime}-L_{1}} \times \frac{1}{\omega-\omega^{\prime}-L_{0}} \rho \mu\right] \\
& =-\frac{1}{\pi} \operatorname{Im} \frac{1}{2 \pi i} \int_{-\infty}^{\infty} d \omega^{\prime} \operatorname{Tr}\left[\mu \cdot \frac{d^{2}}{d \omega^{2}}\left(\frac{1}{\omega-L_{0}}\right) \bar{V}_{1}^{\prime} \frac{1}{\omega^{\prime}-L_{1}} \times \frac{1}{\omega-\omega^{\prime}-L_{0}} \rho \mu\right] \\
& =-\frac{2}{\pi} \operatorname{Im} \frac{1}{2 \pi i} \int_{-\infty}^{\infty} d \omega^{\prime} \operatorname{Tr}\left[\mu \cdot \frac{1}{\left(\omega-L_{0}\right)^{3}} \bar{V}_{1}^{\prime} \frac{1}{\omega^{\prime}-L_{1}} \times \frac{1}{\omega-\omega^{\prime}-L_{0}} \rho \mu\right] .
\end{aligned}
$$

In order to separate the summations over the absorber variables and the bath variables, we first explicitly write the summation indices of the trace in Eq. (32)

$\operatorname{Tr}\left[\mu \cdot \frac{1}{\left(\omega-L_{0}\right)^{3}} \bar{V}_{1}^{\prime} \frac{1}{\omega^{\prime}-L_{1}} \times \frac{1}{\omega-\omega^{\prime}-L_{0}} \rho \mu\right]$

$$
=\operatorname{Tr}\left[\boldsymbol{\mu} \cdot \frac{1}{\left(\omega-L_{0}\right)^{3}} \bar{V}_{1}^{\prime} \frac{1}{\omega^{\prime}-L_{1}} \times \frac{1}{\omega-\omega^{\prime}-L_{0}} \rho^{(b)} \rho^{(a)} \mu\right]_{a, b} .
$$

Then we can carry out the summation over the bath variables of the trace using the fact that there is no contribution from 
the Liouville operator $L^{(b)}$ whenever it acts directly on $\mu$. Therefore, the trace in Eq. (32) can be written as

$\operatorname{Tr}\left[\mu \cdot \frac{1}{\left(\omega-L_{a}\right)^{3}}\left\langle\bar{V}_{1}^{\prime} \frac{1}{\omega^{\prime}-L_{1}}\right\rangle_{b} \frac{1}{\omega-\omega^{\prime}-L_{a}} \rho^{(a)} \mu\right]_{a}$,

where

$$
\left\langle\bar{V}_{1}^{\prime} \frac{1}{\omega^{\prime}-L_{1}}\right\rangle_{b}=\operatorname{Tr}\left[\bar{V}_{1}^{\prime} \frac{1}{\omega^{\prime}-L_{1}} \rho^{(b)}\right]_{b} .
$$

Thus we obtain an expression of $F_{1}(\omega)$ in which the bath variables have also been isolated just as in the expression of $F_{0}(\omega)$

$$
\begin{aligned}
F_{1}(\omega)= & -\frac{2}{\pi} \operatorname{Im} \frac{1}{2 \pi i} \int_{-\infty}^{\infty} d \omega^{\prime} \operatorname{Tr}\left[\mu \cdot \frac{1}{\left(\omega-L_{a}\right)^{3}}\right. \\
& \left.\times\left\langle\bar{V}_{1}^{\prime} \frac{1}{\omega^{\prime}-L_{1}}\right\rangle_{b} \frac{1}{\omega-\omega^{\prime}-L_{a}} \rho^{(a)} \mu\right]_{a} .
\end{aligned}
$$

In comparing Eq. (35) with Eq. (26), we find that the molecular motion introduces complexities in the trace over perturber variables in the calculation of the spectral density.

\section{$E$. The resolvent operator $\left\langle\bar{V}_{1}^{\prime}\left[1 /\left(\omega^{\prime}-L_{1}\right)\right\}\right\rangle_{b}$ and its trajectory average}

The resolvent operator $\left[1 /\left(\omega-L_{1}\right)\right]$ is defined only in the representation constructed from its eigenvectors. Therefore, we have to find the basis in which $L_{1}$ is diagonal. The Liouville operator $L_{1}$ corresponding to the time-independent part is $^{10}$

$$
\begin{aligned}
L_{1} & =\left(V_{\text {aniso }} I^{*}-I V_{\text {aniso }}^{*}\right) / \hbar \\
& =R(r)\left(G_{\text {aniso }} I^{*}-I G_{\text {aniso }}^{*}\right)=R(r) G .
\end{aligned}
$$

We denote by $|\alpha\rangle$ and $G_{\alpha}$, the eigenvectors and eigenvalues, respectively, of the matrix operator $G_{\text {aniso }}$; that is

$$
G_{\text {aniso }}|\alpha\rangle=G_{\alpha}|\alpha\rangle,
$$

or, equivalently, for the Liouville operator $G$ in line space notation

$$
G|\alpha\rangle\left\langle\beta\left|=\left(G_{\alpha}-G_{\beta}\right)\right| \alpha\right\rangle\left\langle\beta\left|\equiv G_{\alpha \beta}\right| \alpha\right\rangle\langle\beta| .
$$

Then, the matrix elements of the Liouville resolvent operator in the representation constructed from its eigenvectors can be written as

$$
\left\langle\alpha \beta^{\dagger}\left|\frac{1}{\omega-L_{1}}\right| \alpha^{\prime} \beta^{\prime \dagger}\right\rangle=\frac{1}{\omega-R(r) G_{\alpha \beta}} \delta_{\alpha \alpha^{\prime}} \delta_{\beta \beta^{\prime}} .
$$

Based on the knowledge about the resolvent operator $\left[1 /\left(\omega-L_{1}\right)\right]$ obtained above, we now discuss the resolvent operator

$$
\left\langle\bar{V}_{1}^{\prime} \frac{1}{\omega^{\prime}-L_{1}}\right\rangle_{b}
$$

in Eq. (35). It is an operator acting on the line space of the absorber molecule $a$. Therefore, in general, it can be expressed as

$$
\begin{aligned}
\left\langle\bar{V}_{1}^{\prime} \frac{1}{\omega^{\prime}-L_{1}}\right\rangle_{b}= & \sum_{i_{1} j_{1}} \sum_{i_{1}^{\prime} j_{1}^{\prime}}\left\langle i_{1} j_{1}^{\dagger}\left|\left\langle\bar{V}_{1}^{\prime} \frac{1}{\omega^{\prime}-L_{1}}\right\rangle_{b}\right| i_{1}^{\prime} j_{1}^{\prime \dagger}\right\rangle \\
& \times\left|i_{1} j_{1}^{\dagger}\right\rangle\left\langle i_{1}^{\prime} j_{1}^{\dagger}\right| .
\end{aligned}
$$

By explicitly writing the summation over the bath molecular variables, its components can be written as

$$
\begin{aligned}
& \left\langle i_{1} j_{1}^{\dagger}\left|\left\langle\bar{V}_{1}^{\prime} \frac{1}{\omega^{\prime}-L_{1}}\right\rangle_{b}\right| i_{1}^{\prime} j_{1}^{\prime \dagger}\right\rangle \\
& \quad=\sum_{i_{2} j_{2}}\left\langle\left(i_{1} j_{1}^{\dagger}\right)\left(i_{2} i_{2}^{\dagger}\right)\left|\bar{V}_{1}^{\prime} \frac{1}{\omega^{\prime}-L_{1}} \rho^{(b)}\right|\left(i_{1}^{\prime} j_{1}^{\prime \dagger}\right)\left(j_{2} j_{2}^{\dagger}\right)\right\rangle .
\end{aligned}
$$

We note that the summation over the bath variables in line space in Eq. (41) includes only the states $\left|i_{2} i_{2}^{\dagger}\right\rangle$ because of the assumption that the bath molecule is not involved in the transition.

Based on our classical ensemble average approximation, the ensemble average over the classical trajectories (indicated by a subscript traj.) is replaced by a weighted integral over $r$ times the parameter $\eta$. Therefore, the trajectoryaveraged component of the resolvent operator $\left\langle\bar{V}_{1}^{\prime}\left[1 /\left(\omega^{\prime}\right.\right.\right.$ $\left.\left.\left.-L_{1}\right)\right]\right\rangle_{b, \text { traj. }}$ can be written as

$$
\begin{aligned}
& \left\langle i_{1} j_{1}^{\dagger}\left|\left\langle\bar{V}_{1}^{\prime} \frac{1}{\omega^{\prime}-L_{1}}\right\rangle_{b, \text { traj. }}\right| i_{1}^{\prime} j_{1}^{\prime \dagger}\right\rangle \\
& =\sum_{i_{2} j_{2}} \sum_{\alpha \beta} \sum_{\alpha^{\prime} \beta^{\prime}}\left\langle\left(i_{1} j_{1}^{\dagger}\right)\left(i_{2} i_{2}^{\dagger}\right) \mid \alpha \beta^{\dagger}\right\rangle\left\langle\alpha \beta^{\dagger}\left|\bar{V}_{1}^{\prime} \frac{1}{\omega^{\prime}-L_{1}}\right| \alpha^{\prime} \beta^{\prime \dagger}\right\rangle_{\text {traj. }}\left\langle\alpha^{\prime} \beta^{\prime \dagger}\left|\rho^{(b)}\right|\left(i_{1}^{\prime} j_{1}^{\prime \dagger}\right)\left(j_{2} j_{2}^{\dagger}\right)\right\rangle \\
& =4 \pi n_{b} \eta \sum_{i_{2 j} / 2} \sum_{\alpha \beta} \sum_{\alpha^{\prime} \beta^{\prime}}\left\langle\left(i_{1} j_{1}^{\dagger}\right)\left(i_{2} i_{2}^{\dagger}\right) \mid \alpha \beta^{\dagger}\right\rangle \int_{r_{\min }}^{\infty} r^{2} d r\left[1-\frac{V_{\text {iso }}(r)}{\frac{1}{2} m v_{\infty}^{2}}\right]^{1 / 2}\left\langle\alpha \beta^{\dagger}\left|\bar{V}_{1}^{\prime} \frac{1}{\omega^{\prime}-R(r) G}\right| \alpha^{\prime} \beta^{\prime \dagger}\right\rangle \\
& \quad \times\left\langle\alpha^{\prime} \beta^{\prime \dagger}\left|\rho^{(b)}\right|\left(i_{1}^{\prime} j_{1}^{\prime \dagger}\right)\left(j_{2} j_{2}^{\dagger}\right)\right\rangle
\end{aligned}
$$

where the factor 


$$
n_{b}\left[1-\frac{V_{\text {iso }}(r)}{\frac{1}{2} m v_{\infty}^{2}}\right]^{1 / 2}
$$

is a statistical weight ${ }^{17}$ and $n_{b}$ is the number density of the bath molecules. We note that in Eq. (42) the lower limit of the integration is not zero but rather the turning point $r_{\min }$ which comes from conservation of energy and consequentially depends on $v_{\infty}$, the relative velocity of two interacting molecules at infinity. Since both the operator $\bar{V}_{1}^{\prime}$ and $r_{\min }$ depend on the velocity $v_{\infty}$, when we carry out the trajectory average, we have also to take into account velocity averaging, assuming a Maxwellian distribution function. However, for simplifying the calculations, the average over $v_{\infty}$ will not be treated exactly, but approximately. We simply replace $v_{\infty}$ by a properly chosen value $\bar{v}_{\infty}$ in the corresponding expressions. Using Eqs. (25) and (39), the matrix elements $\left\langle\alpha \beta^{\dagger}\left|\bar{V}_{1}^{\prime}\left[1 /\left(\omega^{\prime}-L_{1}\right)\right]\right| \alpha^{\prime} \beta^{\prime \dagger}\right\rangle$ in Eq. (42) can be expressed as follows:

$$
\begin{aligned}
\left\langle\alpha \beta^{\dagger}\left|\bar{V}_{1}^{\prime} \frac{1}{\omega^{\prime}-L_{1}}\right| \alpha^{\prime} \beta^{\prime \dagger}\right\rangle= & \left\langle\alpha \beta^{\dagger}\left|\bar{V}_{1}^{\prime}\right| \alpha^{\prime} \beta^{\prime \dagger}\right\rangle \frac{1}{\omega^{\prime}-R(r) G_{\alpha^{\prime} \beta^{\prime}}} \\
= & \left\{\frac{1}{2 m r^{2}}\left[m \bar{v}_{\infty}^{2}-\left(2+r \frac{d}{d r}\right)\left(V_{\text {iso }}(r)+R(r) G_{\alpha \beta}^{+}\right)\right] r \frac{d}{d r} R(r) G_{\alpha \beta} \delta_{\alpha \alpha^{\prime}} \delta_{\beta \beta^{\prime}}\right. \\
& \left.+\frac{1}{2}\left\langle\alpha \beta^{\dagger}\left|\left(\frac{d \theta}{d t}\right)^{2} O^{2} V_{\text {aniso }}\right| \alpha^{\prime} \beta^{\prime \dagger}\right\rangle-\frac{1}{2 m r^{2}}\left\langle\alpha \beta^{\dagger}\left|\left(O V_{\text {aniso }}\right)^{2}\right| \alpha^{\prime} \beta^{\prime \dagger}\right\rangle\right\} \frac{1}{\omega^{\prime}-R(r) G_{\alpha^{\prime} \beta^{\prime}}},
\end{aligned}
$$

where $G_{\alpha \beta}^{+}=\left(G_{\alpha}+G_{\beta}\right)$. It turns out that the contributions to $F_{1}(\omega)$ from the last two terms of Eq. (43) approximately cancel. In fact, if we replace $\frac{1}{2}(d \theta / d t)^{2}$ by $\left(k T / m r^{2}\right)$ and then perform a trace operation, they can be approximated by

$$
\begin{gathered}
{\left[\frac{k T}{2 m r^{2}}\left\langle O^{2} V\right\rangle-\frac{1}{2 m r^{2}}\left\langle(O V)^{2}\right\rangle\right]} \\
\times \frac{1}{\omega^{\prime}-R(r) G_{\alpha^{\prime} \beta^{\prime}}} \delta_{\alpha \alpha^{\prime}} \delta_{\beta \beta^{\prime}},
\end{gathered}
$$

where

$$
\left\langle O^{2} V\right\rangle=\int d \mathbf{r}\left\langle O^{2} V_{\text {aniso }} e^{-V(\mathbf{r}) / k T}\right\rangle_{\Omega}
$$

and $\left\langle(O V)^{2}\right\rangle$ is the mean squared torque defined by

$$
\left\langle(O V)^{2}\right\rangle=\int d \mathbf{r}\left\langle\left(O V_{\text {aniso }}\right)^{2} e^{-V(r) / k T}\right\rangle_{\Omega}
$$

where \langle\rangle$_{\Omega}$ indicates the average over all orientation of two interacting molecules. As is well known classically

$$
k T\left\langle O^{2} V\right\rangle=\left\langle(O V)^{2}\right\rangle
$$

and, therefore, the contributions from these two terms cancel out. Based on the argument given above, we need only to focus on the first term of Eq. (43) and we obtain

$$
\begin{aligned}
\left\langle\alpha \beta^{\dagger}\left|\bar{V}_{1}^{\prime} \frac{1}{\omega^{\prime}-L_{1}}\right| \alpha^{\prime} \beta^{\prime \dagger}\right\rangle \\
=\frac{1}{2 m r^{2}}\left\{m \bar{v}_{\infty}^{2}-\left(2+r \frac{d}{d r}\right)\left(V_{\text {iso }}(r)+R(r) G_{\alpha \beta}^{+}\right)\right\} \\
\quad \times r \frac{d}{d r} R(r) G_{\alpha \beta} \frac{1}{\omega^{\prime}-R(r) G_{\alpha^{\prime} \beta^{\prime}}} \delta_{\alpha \alpha^{\prime}} \delta_{\beta \beta^{\prime}} .
\end{aligned}
$$

However, in the present calculation we are interested in the imaginary part $\operatorname{Im}\left\langle\alpha \beta^{\dagger}\left|\bar{V}^{\prime}\left[1 /\left(\omega^{\prime \prime}-L_{1}\right)\right]\right| \alpha^{\prime} \beta^{\prime \dagger}\right\rangle$ only. With the well-known formal identity

$$
\begin{aligned}
& \lim _{\epsilon \rightarrow 0} \frac{1}{\omega-R(r) G_{\alpha \beta}+i \epsilon} \\
& \quad=P \frac{1}{\omega-R(r) G_{\alpha \beta}}-i \pi \delta\left[\omega-R(r) G_{\alpha \beta}\right],
\end{aligned}
$$

where $P$ indicates the Cauchy principal value of the integral and $\delta$ is a Dirac delta function, it can be expressed as

$$
\begin{aligned}
\operatorname{Im}\left\langle\alpha \beta^{\dagger}\left|\bar{V}_{1}^{\prime} \frac{1}{\omega^{\prime}-L_{1}}\right| \alpha^{\prime} \beta^{\dagger}\right\rangle \\
=-\frac{\pi}{2 m r^{2}}\left\{m \bar{v}_{\infty}^{2}-\left(2+r \frac{d}{d r}\right)\left(V_{\mathrm{iso}}(r)+R(r) G_{\alpha \beta}^{+}\right)\right\} \\
\quad \times r \frac{d}{d r} R(r) G_{\alpha \beta} \delta\left[\omega^{\prime}-R(r) G_{\alpha^{\prime} \beta^{\prime}}\right] \delta_{\alpha \alpha^{\prime}} \delta_{\beta \beta^{\prime}} .
\end{aligned}
$$

Finally, using Eqs. (42) and (49) we can write (see Appendix $\mathbf{B}$ for the details)

$$
\operatorname{Im}\left\langle i_{1} j_{1}^{\dagger}\left|\left\langle\bar{V}_{1}^{\prime} \frac{1}{\omega^{\prime}-L_{1}}\right\rangle_{\text {b.traj. }}\right| i_{1}^{\prime} j_{1}^{\prime \dagger}\right\rangle=\frac{1}{2} \not b_{i_{1} j_{1}, i_{1}^{\prime} j_{1}^{\prime}}\left(\omega^{\prime}\right),
$$

where 


$$
\begin{aligned}
q b_{i_{1} j_{1}, i_{1}^{\prime} j_{1}^{\prime}}(\omega)= & -4 \pi^{2} n_{b} \eta \bar{v}_{\infty}^{2} \sum_{i_{2} j_{2}} \sum_{\alpha \beta}\left\langle\left(i_{1} j_{1}^{\dagger}\right)\left(i_{2} i_{2}^{\dagger}\right) \mid \alpha \beta^{\dagger}\right\rangle \\
& \times\left\{\left[1-\frac{V_{\text {iso }}(r)}{\frac{1}{2} m \bar{v}_{\infty}^{2}}\right]^{1 / 2} \operatorname{Sign}\left[\frac{d R(r)}{d r} G_{\alpha \beta}\right]\right. \\
& \times r\left[1-\frac{1}{m \bar{v}_{\infty}^{2}}\left(2+r \frac{d}{d r}\right)\right. \\
& \left.\left.\times\left(V_{\text {iso }}(r)+R(r) G_{\alpha \beta}^{+}\right)\right]\right\}\left.\right|_{r=r_{\alpha \beta}(\omega)} \\
& \times\left(\alpha \beta^{\dagger}\left|\left(i_{1}^{\prime} j_{1}^{\prime \dagger}\right)\left(j_{2} j_{2}^{\dagger}\right)\right\rangle_{j_{2}} e^{-E_{j_{2}} / k T} / Q_{b}\right.
\end{aligned}
$$

\section{F. The modification of the line shapes}

Using Eq. (B7), the correction term of the spectral density, $F_{1}(\omega)$, can be rewritten as

$$
\begin{aligned}
F_{1}(\omega)= & -\frac{2}{\pi} \operatorname{Im} \frac{1}{2 \pi i} \int_{-\infty}^{\infty} d \omega^{\prime} . \\
& \times \operatorname{Tr}\left[\mu \cdot \frac{1}{\left(\omega-L_{a}\right)^{3}}\left\langle\bar{V}_{1}^{\prime} \frac{1}{\omega^{\prime}-L_{1}}\right\rangle_{b, \text { traj. }}\right. \\
& \left.\times \frac{1}{\omega-\omega^{\prime}-L_{a}} \rho^{(a)} \mu\right]_{a} \\
= & \frac{1}{\pi} \sum_{i j} \sum_{i^{\prime} j^{\prime}} \mu_{i j}^{*} \frac{1}{\left(\omega-\omega_{i j}\right)^{3}} \\
& \times Q l_{i j, i^{\prime} j^{\prime}}\left(\omega-\omega_{i^{\prime} j^{\prime}}\right) \rho_{i^{\prime}} \mu_{i^{\prime} j^{\prime}} .
\end{aligned}
$$

There are a lot of individual $2 b_{i j, i}, j$, functions involved in Eq. (52). However, in practical calculations we can introduce intensity-weighted averaged functions to replace them. The intensity weighted averaged function $\mathscr{Q b}(\omega)$ is defined by

$$
\begin{aligned}
\mathscr{Q b}(\omega) \equiv & \sum_{i j} \sum_{i^{\prime} j^{\prime}} \mu_{i j}^{*} U_{i j, i^{\prime} j^{\prime}}(\omega) \rho_{i^{\prime}} \mu_{i^{\prime} j^{\prime}} / \sum_{i j} \rho_{i}\left|\mu_{i j}\right|^{2} \\
= & -4 \pi^{2} n_{b} \eta \bar{v}_{\infty}^{2} \sum_{\alpha \beta} \sum_{m}\left\langle\alpha\left|\mu_{m}\right| \beta\right)^{*}\left\{\left[1-\frac{V_{i s o}(r)}{\frac{1}{2} m \bar{v}_{\infty}^{-2}}\right]^{1 / 2}\right. \\
& \times \operatorname{Sign}\left[\frac{d R(r)}{d r} G_{\alpha \beta}\right] r\left[1-\frac{1}{m \bar{v}_{\infty}^{2}}\left(2+r \frac{d}{d r}\right)\right. \\
& \left.\left.\times\left(V_{i s o}(r)+R(r) G_{\alpha \beta}^{+}\right)\right]\right\}\left.\right|_{r=r_{\alpha \beta}(\omega)} \\
& \times\left\langle\alpha\left|\rho^{(b)} \rho^{(a)} \mu_{m}\right| \beta\right\rangle / \sum_{i j} \rho_{i}\left|\mu_{i j}\right|^{2}
\end{aligned}
$$

where $r_{\alpha \beta}(\omega)$ are the positive solutions of the equation $\omega-R(r) G_{\alpha \beta}=0$ which are larger than $r_{\min }$. In terms of $\mathscr{Q}(\omega)$, Eq. (52) can approximately be expressed as

$$
F_{1}(\omega)=\frac{1}{\pi} \sum_{i j} \frac{1}{\left(\omega-\omega_{i j}\right)^{3}} Z \zeta\left(\omega-\omega_{i j}\right) \rho_{i}\left|\mu_{i j}\right|^{2}
$$

In analogy to our previous work on band-average line shape functions, ${ }^{10-12}$ we can define the symmetric function

$$
\hat{Q C C}\left(\omega-\omega_{i j}\right) \equiv e^{\hbar\left(\omega-\omega_{i j}\right) / 2 k T} \mathscr{Q C}\left(\omega-\omega_{i j}\right) .
$$

The explicit form of the $\hat{R} C(\omega)$ function is given by

$$
\begin{aligned}
\hat{q} G(\omega)= & -4 \pi^{2} n_{b} \eta \bar{v}_{\infty}^{-2} \sum_{\alpha \beta} \sum_{m}\left\langle\alpha\left|\mu_{m}\right| \beta\right\rangle^{*} e^{\hbar \omega / 2 k T} \\
& \times\left\{[ 1 - \frac { V _ { \text { iso } } ( r ) } { \frac { 1 } { 2 } m v _ { \infty } ^ { - 2 } } ] ^ { 1 / 2 } \operatorname { S i g n } [ \frac { d R ( r ) } { d r } G _ { \alpha \beta } ] r \left[1-\frac{1}{m v_{\infty}^{-2}}\right.\right. \\
& \left.\left.\times\left(2+r \frac{d}{d r}\right)\left(V_{\text {iso }}(r)+R(r) G_{\alpha \beta}^{+}\right)\right]\right\}\left.\right|_{r=r_{\alpha \beta}(\omega)} \\
& \times\left\langle\alpha\left|\rho^{(b)} \rho^{(a)} \mu_{m}\right| \beta\right\rangle / \sum_{i j} \rho_{i}\left|\mu_{i j}\right|^{2} .
\end{aligned}
$$

In terms of this function, the spectral density can be rewritten as

$$
\begin{aligned}
F_{1}(\omega)= & \frac{1}{\pi} \sum_{\omega_{i j}>0} e^{-h\left(\omega-\omega_{i j}\right) / 2 k T}\left\{\frac{1}{\left(\omega-\omega_{i j}\right)^{3}} \hat{\not h}\left(\omega-\omega_{i j}\right)\right. \\
& \left.+\frac{1}{\left(\omega+\omega_{i j}\right)^{3}} \hat{Z} /\left(\omega+\omega_{i j}\right)\right\} \rho_{i}\left|\mu_{i j}\right|^{2}
\end{aligned}
$$

In the present formalism, the absorption coefficients $\alpha(\omega)$ consist of two parts: the main part $\alpha_{0}(\omega)$ related to $F_{0}(\omega)$ and a correction part $\alpha_{1}(\omega)$ related to $F_{1}(\omega)$. The main part of absorption coefficient $\alpha_{0}(\omega)$ obtained in the previous papers, ${ }^{10-12}$ is given by

$$
\begin{aligned}
\alpha_{0}(\omega)= & \frac{4 \pi^{2}}{3 \hbar c} \omega n_{a} \tanh (\hbar \omega / 2 k T)\left[F_{0}(\omega)+F_{0}(-\omega)\right] \\
= & n_{a} \sum_{\omega_{i j}>0} S_{i j} \frac{\omega s h(\hbar \omega / 2 k T)}{\omega_{i j} \operatorname{sh}\left(\hbar \omega_{i j} / 2 k T\right)} \frac{1}{\pi} \\
& \times\left\{\frac{1}{\left(\omega-\omega_{i j}\right)^{2}} \hat{\chi}\left(\omega-\omega_{i j}\right)+\frac{1}{\left(\omega+\omega_{i j}\right)^{2}} \hat{\chi}\left(\omega+\omega_{i j}\right)\right\},
\end{aligned}
$$

where

$$
\begin{aligned}
\hat{\chi}(\omega)= & 4 \pi^{2} n_{b} \omega \sum_{\alpha \beta} \sum_{m}\left\langle\alpha\left|\mu_{m}\right| \beta\right)^{*} \\
& \times e^{-\hbar \omega\left[\left(G_{\alpha}+G_{\beta}\right) /\left(G_{\alpha}-G_{\beta}\right)\right] / 2 k T} \\
& \times\left.\left\{r^{2}\left|\frac{R(r)}{d R(r) / d r}\right| e^{-v_{\text {iso }}(r) / k T}\right\}\right|_{r=r_{\alpha \beta}(\omega)} \\
& \times\left\langle\alpha\left|\rho^{(b)} \rho^{(a)} \mu_{m}\right| \beta\right\rangle / \sum_{i j} \rho_{i}\left|\mu_{i j}\right|^{2},
\end{aligned}
$$


where $r_{\alpha \beta}(\omega)$ are the positive solutions of the equation $\omega-R(r) G_{\alpha \beta}=0$. In Eq. (58), the line strength $S_{i j}$ is defined by the usual expression ${ }^{10-12}$

$$
S_{i j}=\frac{4 \pi^{2} \omega_{i j}}{3 \hbar c Q_{a}} g_{j}\left|\mu_{i j}\right|^{2}\left(e^{-E_{j} / k T}-e^{-E_{i} / k T}\right) .
$$

On the other hand, using the results derived above the correction term can be written in the form

$$
\begin{aligned}
\alpha_{1}(\omega)= & \frac{4 \pi^{2}}{3 \hbar c} \omega n_{a} \tanh (\hbar \omega / 2 k T)\left[F_{1}(\omega)+F_{1}(-\omega)\right] \\
= & n_{a} \sum_{\omega_{i j}>0} S_{i j} \frac{\omega s h(\hbar \omega / 2 k T)}{\omega_{i j} s h\left(\hbar \omega_{i j} / 2 k T\right)} \frac{1}{\pi} \\
& \times\left\{\frac{1}{\left(\omega-\omega_{i j}\right)^{3}} \hat{Q} \ell\left(\omega-\omega_{i j}\right)\right. \\
& +\frac{1}{\left(\omega+\omega_{i j}\right)^{3}} \hat{Q}\left\langle\left(\omega+\omega_{i j}\right)\right\} .
\end{aligned}
$$

In comparing the expressions for $\alpha_{1}(\omega)$ and $\alpha_{0}(\omega)$, we find that they are similar except for the additional power of $\left(\omega-\omega_{i j}\right)$ and $\left(\omega+\omega_{i j}\right)$ in Eq. (61). This is consistent with the expectation that the correction contribution should decrease faster than the leading term as the frequency displacements increases.

\section{NUMERICAL RESULTS FOR THE AT-BROADENED $\nu_{3}$ BAND OF $\mathrm{CO}_{2}$}

As an example, we consider the effect of molecular motion on the wing of the Ar-broadened $\nu_{3}$ band of $\mathrm{CO}_{2}$ for the temperature $T=296 \mathrm{~K}$. In this case, the bath molecule is simply an Ar atom and its internal quantum states need not be considered. In order to simplify the calculations, we model the isotropic potential between $\mathrm{CO}_{2}$ and $\mathrm{Ar}$ by the Lennard-Jones model

$$
V_{\text {iso }}(r)=4 \epsilon\left[\left(\frac{\sigma}{r}\right)^{12}-\left(\frac{\sigma}{r}\right)^{6}\right]
$$

The expression of the anisotropic potential, Eq. (16) is the product of the functions

$$
R(r)=4 \epsilon\left[R_{2}\left(\frac{\sigma}{r}\right)^{12}-A_{2}\left(\frac{\sigma}{r}\right)^{6}\right]
$$

and

$$
G_{\text {aniso }}=P_{2}(\cos \theta),
$$

where $\theta$ is the angle between $\mathbf{r}$ and the $\mathrm{CO}_{2}$ molecular axis, and $R_{2}$ and $A_{2}$ are adjustable parameters. We first adopt the same values used by Boissoles et al. ${ }^{14}$ with $\sigma=3.91 \AA$, $\epsilon / k$ $=153 \mathrm{~K}, R_{2}=1.09$ and $A_{2}=0.266$. For the frequency range of interest in the present calculations, $2400-2580 \mathrm{~cm}^{-1}$, we consider the contributions from the $\nu_{3}$ band of $\mathrm{CO}_{2}$ only and exclude any contributions from other bands. From the HITRAN data base, ${ }^{18}$ there are 109 lines listed for this band and the maximum value of the rotational quantum numbers among them is 108 . Although all of them are included in the calculations, the diagonization of the anisotropic interaction matrix whose size depends on how many states are included in the complete set remains tractable.

The formula used by Boissoles et al. ${ }^{14}$ to calculate the band-averaged line shape function of $\nu_{3}$ band of $\mathrm{CO}_{2}$ is similar to Eq. (59). However, they introduced an energetically corrected function in their formalism. This correction corresponds to rewriting the spectral density in a more symmetric form $^{19}$

$$
F_{0}(\omega)=\frac{1}{\pi} \operatorname{Re} \int_{0}^{\infty} e^{i \omega t} \operatorname{Tr}\left\{\mu \sqrt{\rho} \cdot e^{-i L t} \sqrt{\rho} \mu\right\} d t .
$$

Consequently, the expression of the band-averaged line shape function $\hat{\chi}(\omega)$ is given by

$$
\begin{aligned}
\hat{\chi}(\omega)= & 4 \pi^{2} n_{b} \omega \sum_{\alpha \beta} \sum_{m}\left|\left\langle\alpha\left|\sqrt{\rho} \mu_{m}\right| \beta\right\rangle\right|^{2} \\
& \times e^{-\hbar \omega\left[\left(G_{\alpha}+G_{\beta}\right) /\left(G_{\alpha}-G_{\beta}\right)\right] / 2 k T} \\
& \times\left.\left\{r^{2}\left|\frac{R(r)}{d R(r) / d r}\right| e^{-V_{\text {iso }}(r) / k T}\right\}\right|_{r=r_{\alpha \beta}(\omega)} \\
& / \sum_{i j} \rho_{i}\left|\mu_{i j}\right|^{2} .
\end{aligned}
$$

Similarly, we follow the same energetically corrected procedure to calculate $\hat{t},(\omega)$. As we mentioned previously, in the present calculations we do not treat the velocity average exactly. Since the Maxwellian distribution function has a sharp maximum at $v_{\max }=\sqrt{2 k T / m}$, we choose $\bar{v}_{\infty}=v_{\max }$ in the trajectory average calculations. With respect to the turning points $r_{\min }$, we assume that they are determined by the isotropic potential only and that the effect from the anisotropic potential is averaged out. In the present calculation, we simply use the equation, $\frac{1}{2} m \bar{v}_{\infty}^{2}-V_{\text {iso }}\left(r_{\text {min }}\right)=0$ to determine the turning point $r_{\min }$. Of course, more sophisticated methods could be used to deal with the velocity averaging and the determination of the turning points, however, we do not discuss them in the present paper. Thus the formula used for the numerical calculations for $\hat{b}(\omega)$ is

$$
\begin{aligned}
\hat{Z} \hat{l}(\omega)= & -8 \pi^{2} n_{b} \eta \frac{k T}{m} \sum_{\alpha \beta} \sum_{m}\left|\left\langle\alpha\left|\sqrt{\rho} \mu_{m}\right| \beta\right\rangle\right|^{2} e^{\hbar \omega / 2 k T} \\
& \times\left\{\left[1-\frac{V_{\text {iso }}(r)}{k T}\right]^{1 / 2} \operatorname{Sign}\left[\frac{d R(r)}{d r} G_{\alpha \beta}\right]\right. \\
& \times r\left[1-\frac{1}{2 k T}\left(2+r \frac{d}{d r}\right)\left(V_{\text {iso }}(r)\right.\right. \\
& \left.\left.\left.+R(r) G_{\alpha \beta}^{+}\right)\right]\right\}\left.\right|_{r=r_{\alpha \beta}(\omega)} / \sum_{i j} \rho_{i}\left|\mu_{i j}\right|^{2}
\end{aligned}
$$

As mentioned previously, the value of the parameter $\eta$ introduced to make the classical trajectory average approximation consistent with the closest region approximation is unknown. Using a classical model (see Appendix C), we estimate $\eta=0.3$ which we use in the present calculations. 


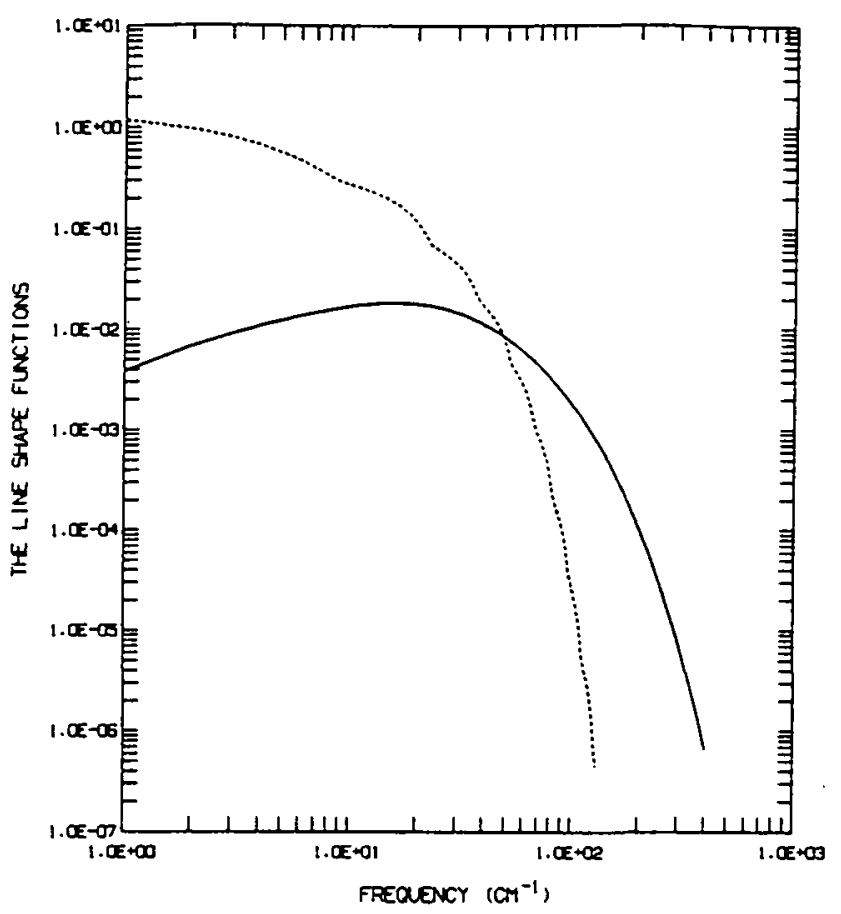

FGG. 1. The band-averaged line shape function $\dot{x}(\omega)$ in units of $\mathrm{cm}^{-1} \mathrm{~atm}^{-1}$ (solid curve) and the correction to the band-averaged line shape function, $\forall /(\omega) / \omega$ with $\eta=0.3$ (dotted curve), as functions of frequency $\omega$ (in units of $\mathrm{cm}^{-1}$ ) calculated for $T=296 \mathrm{~K}$.

The calculated band averaged line shape function $\hat{\chi}(\omega)$ and the calculated correction of the band averaged line shape function $\hat{Q} \ell(\omega)$ are shown in Fig. 1. In this figure we present $\hat{Q} G(\omega) / \omega$ instead of $\hat{Q} b(\omega)$ since not only $\hat{Q} b(\omega) / \omega$ has the same dimension as $\hat{\chi}(\omega)$, but also the comparison between them makes more sense. The frequency at which $\hat{\imath} b(\omega) / \omega$ becomes comparable to $\hat{\chi}(\omega)$ depends on the short-range anisotropic potential and on the value of the parameter $\eta$, in the present case, this occurs around $45 \mathrm{~cm}^{-1}$. For smaller frequencies, $\hat{\imath} b(\omega) / \omega$ exceeds $\hat{\chi}(\omega)$ implying that the quasistatic approximation breaks down and the effects of molecular motion must be taken into account. As shown in the Fig. 1, $\hat{Z} 6(\omega) / \omega$ falls off faster than $\hat{x}(\omega)$ as the frequency increases and becomes negligible beyond $80 \mathrm{~cm}^{-1}$ which validates the quasistatic approximation for this frequency region.

The calculated contributions of absorption coefficients from the main part $F_{0}(\omega)$ which corresponds to Boissoles et al.'s results ${ }^{14}$ are shown in Fig. 2. The total absorption coefficients are also shown in Fig. 2 together with the results obtained using a Lorentzian line shape and the experimental results. ${ }^{14}$ In order to investigate how sensitive these results are to the choice of the potential parameters, we have repeated the calculations varying the parameters $\sigma, \epsilon / k, R_{2}$, and $A_{2}$ in Eqs. (62) and (63). As expected the results depend sensitively on $\sigma$ but less so on $\epsilon / k$ : small changes in the relative weighting of the long- and short-range parts $A_{2}$ and $R_{2}$ can affect the magnitude. We obtained the agreement with the experimental data shown in Fig. 3 using the values: $\sigma=3.2 \AA$, $\epsilon / k=100 \mathrm{~K}, R_{2}=1.22$, and $A_{2}=0.35$. Finally, we note that the comparisons discussed above are based on calculations made with a simplified one-component anisotropic

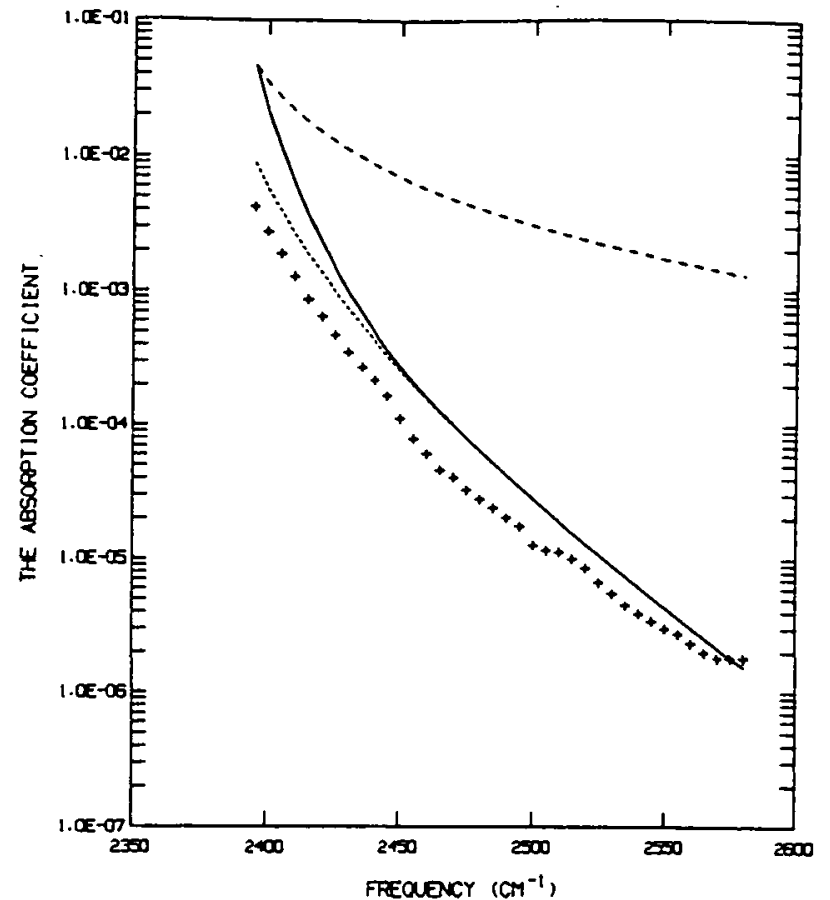

FIG. 2. The absorption coefficient $\alpha(\omega)$ (in units of $\mathrm{cm}^{2}$ molecule $\mathrm{atm}^{-1}$ ) vs the frequency $\omega$ (in units of $\mathrm{cm}^{-1}$ ) calculated for $T=296 \mathrm{~K}$. The curve $\cdots$ is the contribution from the band-averaged line shape function and - is the total. Also shown are the results calculated using a Lorentrian line shape denoted by .-- and the experimental data denoted by + , respectively.

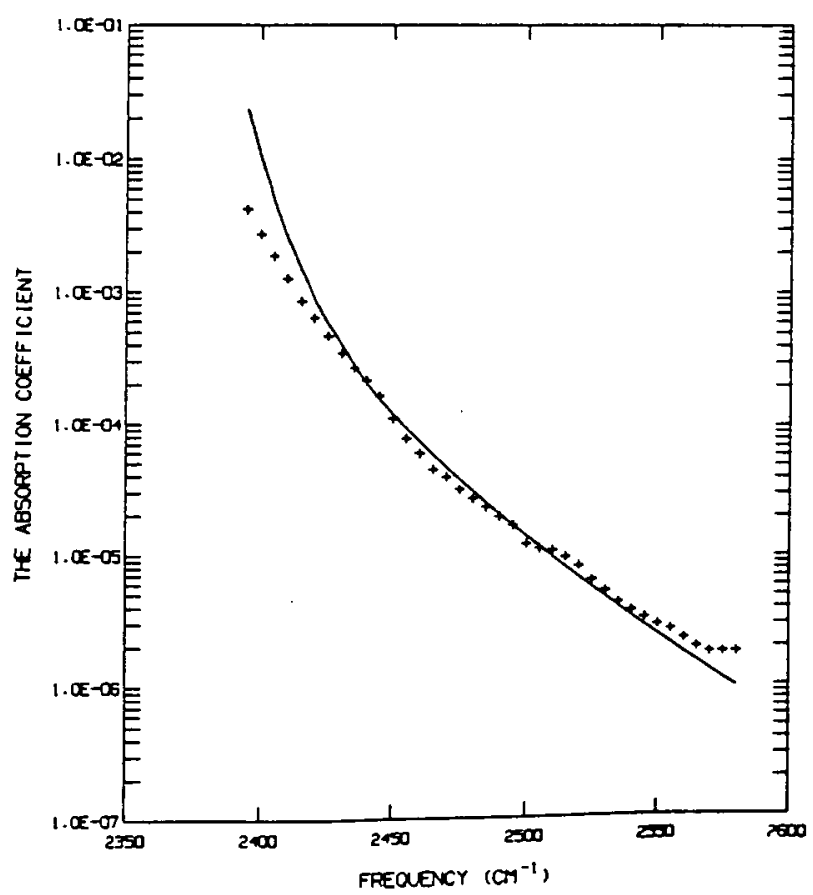

FIG. 3. Comparison between the present theoretical results calculuted using the modified potential parameters and the experimental dex 
potential. Before accurate quantitative comparisons can be made, a more realistic multicomponent potential must be used.

\section{DISCUSSION AND CONCLUSIONS}

As a result of the present work, several conclusions can be drawn. First, the effects of molecular motion significantly affect the quasistatic line shape for displacements $\Delta \omega$ less than $50 \mathrm{~cm}^{-1}$ from the line centers. As noted above for smaller displacements, the correction term can exceed the quasistatic contributions; however, in this region $1 / \tau_{c}<\Delta \omega=1 / \tau$ where $\tau_{c}$ and $\tau$ are time between collision and the duration, respectively, both the quasistatic and the impact theories break down. As a result, the corrections for molecular motion will be more important nearer the band origin or in the microwindows between the lines ${ }^{20}$ than in the windows between vibration-rotational bands. Second, the correction depend on the interaction potential, and because the motion is greater for higher temperatures or for lighter molecules, one would expect larger corrections in these cases. Conversely, by varying the parameters in the potential in order to obtain agreement between theory and experiment, one can infer these parameters. However, we note that one of the drawbacks of the present quasistatic formalism is that only a simplified form of the anisotropic potential having one spherical harmonic can be incorporated. Further work to include more general potentials is needed before realistic potential-energy parameters can be accurately determined.
Finally, although the present work is a first attempt to consider the molecular motion a number of simplifying assumptions have been introduced in the calculations; nevertheless to take into account of the correction of the quasistatic formalism due to molecular motion is necessary in the nearwing region and this becomes a tractable problem especially for some simple interacting systems.

Some refinements of the approximations can be incorporated in the formalism. For example, one can improve the trajectory average and avoid the introduction of the parameter $\eta$. Because a main uncertainty in the present calculation is related to the interaction potential, there are two challenges facing us. One is to find a more reliable but still tractable potential models to represent the interaction. The other is to find a theoretical method to calculate the resolvent operator corresponding to more realistic anisotropic potentials which consists of more than one spherical harmonic component. Further work is needed in this direction.

\section{ACKNOWLEDGMENTS}

This work was supported in part by the Department of Energy Interagency Agreement under the Atmospheric Radiation Measurement Program and by a NASA grant under the Venus Data Analysis Program. We would like to thank the National Energy Research Supercomputer Center (Livermore, CA) for computer time and facilities provides and to thank Dr. Boissoles for helpful discussions conceming the quasistatic calculations of the $\nu_{3}$ band of $\mathrm{CO}_{2}$.

\section{APPENDIX A}

The first-order term of the spectral density is

$$
F_{1}(\omega)=\frac{1}{2 \pi} \int_{-\infty}^{\infty} e^{i \omega t} \operatorname{Tr}\left\{\rho \mu \cdot i e^{i L t}\left[\int_{0}^{t} V_{1}^{l}\left(t^{\prime}\right) d t^{\prime}, \mu\right]\right\} d t=\frac{1}{\pi} \operatorname{Re} \int_{0}^{\infty} e^{i \omega t} \operatorname{Tr}\left\{\rho \mu \cdot i e^{i L t}\left[\int_{0}^{t} V_{1}^{I}\left(t^{\prime}\right) d t^{\prime}, \mu\right]\right\} d t
$$

In the step of deriving Eq. (A1), we have used the fact that

$$
\left\{i e^{-i L t}\left[\int_{0}^{-s} V_{1}^{l}\left(t^{\prime}\right) d t^{\prime}, \mu\right]\right\}^{*}=-i e^{i L t}\left[\int_{0}^{-t} V_{1}^{l}\left(t^{\prime}\right)^{*} d t^{\prime}, \mu\right]=i e^{i L t}\left[\int_{0}^{t} V_{1}^{l}\left(-t^{\prime}\right)^{*} d t^{\prime}, \mu\right]=i e^{i L t}\left[\int_{0}^{t} V_{1}^{l}\left(t^{\prime}\right) d t^{\prime}, \mu\right],
$$

since $V_{1}(t)$ is an even function as mentioned in the text. Then,

$$
F_{1}(\omega)=-\frac{1}{\pi} \operatorname{Re} \int_{0}^{\infty} e^{i \omega t} \operatorname{Tr}\left\{\mu \cdot\left[i \int_{0}^{t} V_{1}^{\prime}\left(t^{\prime}\right) d t^{\prime}, e^{-i L t} \rho \mu\right]\right\} d t=\frac{1}{\pi} \operatorname{Im} \int_{0}^{\infty} e^{i \omega t} \operatorname{Tr}\left\{\mu \cdot\left[\int_{0}^{t} V_{1}^{\prime}\left(t^{\prime}\right) d t^{\prime}, e^{-i L t} \rho \mu\right]\right\} d t
$$

The integration of Eq. (A2) can expressed as follows:

$$
\begin{aligned}
\int_{0}^{\infty} d t e^{i \omega t}\left[\int_{0}^{t} V_{1}^{\prime}\left(t^{\prime}\right) d t^{\prime}, e^{-i L t} \rho \mu\right] & =\int_{0}^{\infty} d t\left[\int_{0}^{t} V_{1}^{\prime}\left(t^{\prime}\right) d t^{\prime}, e^{i(\omega-L) t} \rho \mu\right]=\int_{0}^{\infty}\left[\int_{0}^{t} V_{1}^{\prime}\left(t^{\prime}\right) d t^{\prime}, d \int_{0}^{t} e^{i(\omega-L) t^{\prime}} d t^{\prime} \rho \mu\right] \\
& \left.=\left[\int_{0}^{t} V_{1}^{\prime}\left(t^{\prime}\right) d t^{\prime}, \int_{0}^{t} e^{i(\omega-L) t^{\prime}} d t^{\prime} \rho \mu\right]\right]_{0}^{\infty}-\int_{0}^{\infty} d t\left[V_{1}^{\prime}(t), \int_{0}^{t} e^{i(\omega-L) t^{\prime}} d t^{\prime} \rho \mu\right] \\
& =\left[\int_{0}^{\infty} V_{1}^{\prime}\left(t^{\prime}\right) d t^{\prime}, \int_{0}^{\infty} e^{i(\omega-L) t^{\prime}} d t^{\prime} \rho \mu\right]
\end{aligned}
$$




$$
\begin{aligned}
& +i \int_{0}^{\infty} d t\left[V_{1}^{t}(t),\left.e^{i(\omega-L) t^{\prime}}\right|_{0} ^{t} \frac{1}{\omega-L} \rho \mu\right] \\
= & i \int_{0}^{\infty} d t e^{i \omega t}\left[e^{-i L t} V_{1}(t), e^{-i L t} \frac{1}{\omega-L} \rho \mu\right] \\
= & i \int_{0}^{\infty} d t e^{i(\omega-L) t}\left[V_{1}(t), \frac{1}{\omega-L} \rho \mu\right]=i \int_{0}^{\infty} d t e^{i(\omega-L) t} \bar{V}_{1}(t) \frac{1}{\omega-L} \rho \mu,
\end{aligned}
$$

where $\bar{V}_{1}(t)$ is a Liouville operator corresponding to $V_{1}(t)$. With Eq. (A3), we rewrite the first-order term as

$$
F_{1}(\omega)=\frac{1}{\pi} \operatorname{Re} \int_{0}^{\infty} e^{i \omega t} \operatorname{Tr}\left\{\mu \cdot e^{-i L t} \bar{V}_{1}(t) \frac{1}{\omega-L} \rho \mu\right\} d t .
$$

To further simplify the calculations, we introduce another approximation in Eq. (A4). As discussed in the text, the part of $V_{1}(t)$, i.e., the term of $\frac{1}{2} t^{2}\left(d^{2} r / d t^{2}\right)[d R(r) / d r] G_{\text {aniso }}$, which contributes to $F_{1}(\omega)$ only, commutes with the time-independent interaction $V$; therefore, $e^{-i L^{t}} V_{1}(t) \approx e^{-i L_{0} t} V_{1}(t)$. With this fact and keeping in mind that we expect the effects due to the molecular motion to be significant only in near wings, we will approximate the Liouville operator $e^{-i L t}$ by its main part $e^{-i L_{0^{t}}}$ in Eq. (A4). Then we obtain the simple formula

$$
F_{1}(\omega)=\frac{1}{\pi} \operatorname{Re} \int_{0}^{\infty} e^{i \omega t} \operatorname{Tr}\left\{\mu \cdot e^{-i L_{0} t} \tilde{V}_{1}(t) \frac{1}{\omega-L} \rho \mu\right\} d t
$$

\section{APPENDIX B}

With Eqs. (42) and (49), the trajectory averaged component of the resolvent operator $\left\langle\bar{V}_{1}^{\prime}\left[1 /\left(\omega^{\prime}-L_{1}\right)\right]\right\rangle_{b}$ can be expressed

$$
\begin{aligned}
& \operatorname{Im}\left\langle i_{1} j_{1}^{\dagger}\left|\left\langle\tilde{V}_{1}^{\prime} \frac{1}{\omega^{\prime}-L_{1}}\right\rangle_{b, \text { traj. }}\right| i_{1}^{\prime} j_{1}^{\prime \dagger}\right\rangle=-2 \pi^{2} n_{b} \eta \dot{v}_{\infty}^{2} \sum_{i_{2} / 2} \sum_{\alpha \beta} \int_{r_{\min }}^{\infty} r d r\left\langle\left(i_{1} j_{1}^{\dagger}\right)\left(i_{2} i_{2}^{\dagger}\right) \mid \alpha \beta^{\dagger}\right\rangle\left[1-\frac{V_{i s o}(r)}{\frac{1}{2} m \bar{v}_{\infty}^{-2}}\right]^{1 / 2} \\
& \times\left\{1-\frac{1}{m \bar{v}_{\infty}^{2}}\left(2+r \frac{d}{d r}\right)\left(V_{\text {iso }}(r)+R(r) G_{\alpha \beta}^{+}\right)\right\} \frac{d}{d r} R(r) G_{\alpha \beta} \delta\left[\omega^{\prime}-R(r) G_{\alpha \beta}\right] \\
& \times\left\langle\alpha \beta^{\dagger} \mid\left(i_{1}^{\prime} j_{1}^{\prime \dagger}\right)\left(j_{2} j_{2}^{\dagger}\right)\right\rangle g_{j 2} e^{-E_{j 2} / k r} / Q_{b},
\end{aligned}
$$

where $g_{j}$ are the degeneracy factors due to nuclear spin, $E_{j}$ are the energies associated with the state $|j\rangle$, and $Q_{b}$ is the partition function for the bath molecules. By carrying out the integration with the help of the $\delta$ function, we obtain

$$
\begin{aligned}
& \operatorname{Im}\left\langle i_{1} j_{1}^{\dagger}\left|\left\langle\bar{V}_{1}^{\prime} \frac{1}{\omega^{\prime}-L_{1}}\right\rangle_{b, \text { raj. }}\right| i_{1}^{\prime} j_{1}^{\dagger}\right\rangle=-2 \pi^{2} n_{b} \eta \tilde{v}_{\infty}^{2} \sum_{i_{2} j_{2}} \sum_{\alpha \beta}\left\langle\left(i_{1} j_{1}^{\dagger}\right)\left(i_{2} i_{2}^{\dagger}\right) \mid \alpha \beta^{\dagger}\right\rangle \\
& \times\left\{\left[1-\frac{V_{\mathrm{iso}}(r)}{\frac{1}{2} m \bar{v}_{\infty}^{-2}}\right]^{1 / 2} \operatorname{Sign}\left[\frac{d R(r)}{d r} G_{\alpha \beta}\right]\right. \\
& \left.\times r\left[1-\frac{1}{m \tilde{v}_{\infty}^{2}}\left(2+r \frac{d}{d r}\right)\left(V_{\mathrm{iso}}(r)+R(r) G_{\alpha \beta}^{+}\right)\right]\right\}\left.\right|_{r=r_{\alpha \beta}\left(\omega^{\prime}\right)} \\
& \times\left\langle\alpha \beta^{\dagger} \mid\left(i_{1}^{\prime} j_{1}^{\prime \dagger}\right)\left(j_{2} j_{2}^{\dagger}\right)\right\rangle g_{j 2} e^{-E_{j 2} / k T} / Q_{b}, \\
& \equiv \frac{1}{2} \mathscr{Q}_{i_{1} j_{1}, i_{1}^{\prime} j_{1}^{\prime}}\left(\omega^{\prime}\right) \text {, }
\end{aligned}
$$

where $r_{\alpha \beta}\left(\omega^{\prime}\right)$ are the positive solutions of the equation $\omega^{\prime}-R(r) G_{\alpha \beta}=0$ which are larger than $r_{\min }$. In the above expression,

$$
\begin{aligned}
2 b_{i_{1} j_{1}, i_{1}^{\prime} j_{1}^{\prime}}\left(\omega^{\prime}\right)= & -4 \pi^{2} n_{b} \eta \bar{v}_{\infty}^{2} \sum_{i_{2 j} j_{2}} \sum_{\alpha \beta}\left\langle\left(i_{1} j_{1}^{\dagger}\right)\left(i_{2} i_{2}^{\dagger}\right) \mid \alpha \beta^{\dagger}\right\rangle\left\langle[ 1 - \frac { V _ { \mathrm { iso } } ( r ) } { \frac { 1 } { 2 } m \overline { v } _ { \infty } ^ { 2 } } ] ^ { 1 / 2 } \operatorname { S i g n } [ \frac { d R ( r ) } { d r } G _ { \alpha \beta } ] r \left[ 1-\frac{1}{m \bar{v}_{\infty}^{2}}\left(2+r \frac{d}{d r}\right)\right.\right. \\
& \times\left.\left(V_{\mathrm{iso}}(r)+R(r) G_{\alpha \beta}^{+}\right)\right|_{r=r_{\alpha \beta}\left(\omega^{\prime}\right)}\left\langle\alpha \beta^{\dagger} \mid\left(i_{1}^{\prime} j_{1}^{\prime \dagger}\right)\left(j_{2} j_{2}^{\dagger}\right)\right\rangle g_{j 2} e^{-\varepsilon_{j 2} / k r} / Q_{b} .
\end{aligned}
$$


With Eqs. (35) and (B2), the correction term of the spectral density, $F_{1}(\omega)$, can be rewritten as

$$
\begin{aligned}
F_{1}(\omega) & =-\frac{2}{\pi} \operatorname{Im} \frac{1}{2 \pi i} \int_{-\infty}^{\infty} d \omega^{\prime} \operatorname{Tr}\left[\mu \cdot \frac{1}{\left(\omega-L_{a}\right)^{3}}\left\langle\bar{V}_{1}^{\prime} \frac{1}{\omega^{\prime}-L_{1}}\right)_{b, \text { traj. }} \frac{1}{\omega-\omega^{\prime}-L_{a}} \rho^{(a)} \mu\right]_{a} \\
& =-\frac{1}{\pi} \sum_{i j} \sum_{i^{\prime} j^{\prime}}\langle j|\mu| i\rangle \cdot \frac{1}{\left(\omega-\omega_{i j}\right)^{3}} \frac{1}{2 \pi i} \int_{-\infty}^{\infty} d \omega^{\prime} \mathscr{Q} b_{i, i^{\prime} j^{\prime}}\left(\omega^{\prime}\right) \frac{1}{\omega-\omega^{\prime}-\omega_{i^{\prime} j^{\prime}}} \rho_{i^{\prime}}\left\langle i^{\prime}|\mu| j^{\prime}\right\rangle \\
& =\frac{1}{\pi} \sum_{i j} \sum_{i^{\prime} j^{\prime}}\langle j|\mu| i\rangle \frac{1}{\left(\omega-\omega_{i j}\right)^{3}} \mathscr{Q} b_{i j, i^{\prime} j^{\prime}}\left(\omega-\omega_{i{ }^{\prime} j^{\prime}}\right) \rho_{i^{\prime}}\left\langle i^{\prime}|\mu| j^{\prime}\right\rangle .
\end{aligned}
$$

Using the reduced matrix elements,

$$
\left\langle i\left|\mu_{m}\right| j\right\rangle=\left[\frac{1}{(2 i+1)}\right]^{1 / 2} C\left(j 1 i ; m_{j} m m_{i}\right) \mu_{i j},
$$

and reduced functions, $2 b_{i j, i^{\prime} j}^{\text {redu }}(\omega)$, defined by

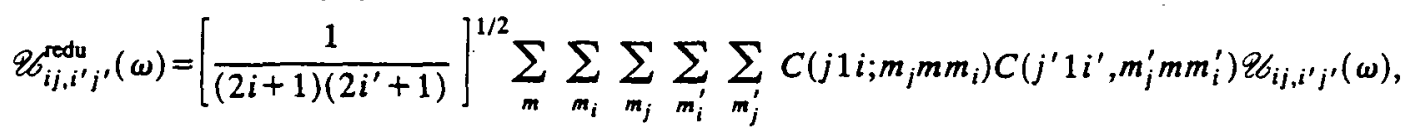

we complete the summation over the magnetic quantum numbers and obtain

$$
\begin{aligned}
F_{1}(\omega)= & \frac{1}{\pi} \sum_{i j} \sum_{i^{\prime} j^{\prime}} \mu_{i j}^{*} \frac{1}{\left(\omega-\omega_{i j}\right)^{3}} \\
& \times Q \zeta_{i j, i^{\prime} j^{\prime}}^{\text {edu }}\left(\omega-\omega_{i^{\prime} j^{\prime}}\right) \rho_{i^{\prime}} \mu_{i^{\prime} j^{\prime}},
\end{aligned}
$$

where the summation indices exclude their magnetic quantum numbers. For simplicity, since we will use these reduced forms instead of the original ones in the results that follow, we will omit the superscript redu.

\section{APPENDIX C}

In general for a specified relative velocity at infinity, $v_{\infty}$, the trajectory is determined by the impact parameter $b$ and the interaction potential $V(\mathbf{r})$, and the relative position of molecules along the trajectory $s(t)$ is a function of time whose zero has been chosen in the present work as the time of closest approach. In order to calculate averages of the functions of interest over all trajectories, one has to average over $b$ and $s(t)$. However, the functions of interest are usually expressed in terms of the intermolecular separation $r$ and, therefore, the dynamic variables $b$ and $s(t)$ are not appropriate and have to be transformed to spherical coordinates. Assuming that the trajectory is mainly determined by the isotropic potential, we have

$$
\frac{d r}{d t}=v_{\infty}\left[1-\frac{2 V_{\text {iso }}(r)}{m v_{\infty}^{2}}-\frac{b^{2}}{r^{2}}\right]^{1 / 2} .
$$

For a specified $r$ the maximum impact parameter is given by

$$
b_{\max }(r)=r\left[1-\frac{2 V_{\text {iso }}(r)}{m v_{\infty}^{2}}\right]^{1 / 2} \text {. }
$$

With the straight line trajectory approximation, the integral of a function $F(r)$ over all trajectories can be expressed as

$$
\begin{aligned}
I & =2 \pi \int_{0}^{\infty} b d b \int_{-\infty}^{\infty} F(r) v_{\infty} d t, \\
& =4 \pi \int_{r_{\min }}^{\infty} F(r) d r \int_{0}^{b_{\max }(r)}\left[1-\frac{2 V_{\mathrm{iso}}(r)}{m v_{\infty}^{2}}-\frac{b^{2}}{r^{2}}\right]^{-1 / 2} b d b, \\
& =4 \pi \int_{r_{\min }}^{\infty}\left[1-\frac{2 V_{\text {iso }}(r)}{m v_{\infty}^{2}}\right]^{1 / 2} F(r) r^{2} d r,
\end{aligned}
$$

where $r_{\min }$ is given by

$$
\frac{1}{2} m v_{\infty}^{2}-V_{\text {iso }}\left(r_{\min }\right)=0 .
$$

However, based on the closest region approximation the contribution to the integral $I$ comes mainly from the region near the turning point of trajectories and, in fact, the function $F(r)$ has been expressed in terms of an expansion around this point. For consistency, this implies that a cutoff has to be enforced on the integration along trajectories. One method to introduce the cutoff is to replace 0 , the lower limit of the integration over $b$ in Eq. (C3), by $b_{\min }(r)$ and to assume that

$$
b_{\min }(r)=b_{\max }(r) \cos \theta_{0},
$$

where $\theta_{0}$ is a parameter. Then, one is able to recast Eq. (C3) as

$$
\begin{aligned}
I & =4 \pi \int_{r_{\min }}^{\infty} F(r) d r \int_{b_{\min }(r)}^{b_{\max }(r)}\left[1-\frac{2 V_{\mathrm{iso}}(r)}{m v_{\infty}^{2}}-\frac{b^{2}}{r^{2}}\right]^{-1 / 2} b d b, \\
& =4 \pi \eta \int_{r_{\min }}^{\infty}\left[1-\frac{2 V_{\mathrm{iso}}(r)}{m v_{\infty}^{2}}\right]^{1 / 2} F(r) r^{2} d r,
\end{aligned}
$$

where the parameter $\eta$ finally used in the text is defined by

$$
\eta=\sin \theta_{0}=\left[1-\frac{b_{\min }^{2}}{b_{\max }^{2}}\right]^{1 / 2} .
$$


In order to estimate the parameter $\eta$, we consider a straight line trajectory with the closest distance denoted by $r_{0}(=b)$. In the present case, the function $F(r)$ of interest is $V_{1}^{\prime}=\frac{1}{2}\left(d^{2} r / d t^{2}\right)(\partial / \partial r) V_{\text {aniso }}(r)$. If we assume that around the distance of closest approach the repulsive part of the anisotropic potential dominates, then $F(r) \propto r^{-16}$ and its magnitude drops to $1 / 2$ the maximum value for $s \approx 0.3 r_{0}$. With this assumption, the interaction takes place along the trajectory between $-0.3 r_{0}<s<+0.3 r_{0}$. It corresponds to $b_{\min }=r_{0}$ and $b_{\max }=\left[1+\left(s^{2} / r_{0}^{2}\right)\right]^{1 / 2} r_{0}$. Therefore, one is able to estimate the parameter $\eta=\left(s / b_{\text {max }}\right) \simeq 0.3$ which we use in the calculations. In Eq. (C6) and consequently in Eq. (67) the parameter $\eta$ is simply a scale parameter and it is easy to calculate results which correspond to different values of $\eta$. For example, the correction contribution is comparabie to the leading contribution at about $27 \mathrm{~cm}^{-1}$ for $\eta=0.1$ while this occurs at $45 \mathrm{~cm}^{-1}$ for $\eta=0.3$. Although there is considerable uncertainty in the potential for small separations and arbitrariness in the cutoff discussed above and thus in the value of $\eta$, we note that the general conclusion that molecular motion is important for the near wings of the lines but can be ignored in the far-wing region does not depend on the value of $\eta$ assumed.
'J. H. Van Vleck and D. L. Huber, Rev. Mod. Phys. 49, 939 (1977).

${ }^{2}$ P. W. Anderson, Phys. Rev. 76, 647 (1949).

${ }^{3}$ C. J. Tsao and B. Curmutte, J. Quant. Spectrose. Radiat. Transfer 2, 41 (1962).

${ }^{4}$ P. W. Rosenkranz, J. Chem. Phys. 83, 6139 (1985).

${ }^{5}$ P. W. Rosenkranz, J. Chem. Phys. 87, 163 (1987).

${ }^{6}$ U. Fano, Phys. Rev. 131, 259 (1963).

'D. E. Burch and D. A. Gryvnak, AFGL-TR-0054, 1979; D. E. Burch, SPIE Proc. 277, 28 (1981); D. E. Burch and R. L. Alt, AFGL-TR-84-0128, 1984; D. E. Burch, AFGL-TR-85-0036, 1985.

${ }^{8}$ C. Boulet, J. Boissoles, and D. Robert, J. Chem. Phys. 89,625 (1988).

${ }^{9} \mathrm{Q}$. Ma and R. H. Tipping, J. Chem. Phys. 93, 7066 (1990).

${ }^{10} \mathrm{Q}$. Ma and R. H. Tipping, J. Chem. Phys. 95, 6290 (1991).

"Q. Ma and R. H. Tipping, J. Chem. Phys. 96, 8655 (1992).

${ }^{12} \mathrm{Q}$. Ma and R. H. Tipping, J. Chem. Phys. 97, 818 (1992).

${ }^{13} \mathrm{Q}$. Ma and R. H. Tipping, J. Chem. Phys. (to be published).

${ }^{14}$ J. Boissoles, V. Menoux, R. Le Doucen, C. Bouleh, and D. Rober, J. Chem. Phys. 91, 2163 (1989).

${ }^{15} \mathrm{R}$. H. Tipping and Q. Ma, Atmos. Res. (to be published).

${ }^{16} \mathrm{G}$. Birmbaum, in Spectral Line Shapes, AIP Conference Proceedings (AIP, New York, 1990).

${ }^{17}$ C. Boulet and D. Robert, J. Chem. Phys. 77, 4288 (1982).

${ }^{18}$ L. S. Rothman, R. R. Gamache, R. H. Tipping, C. P. Rinsland, M. A. H. Smith, D. Chris Benner, V. Malathy Devi, J.-M. Flaud, C. Camy-Peyret, A. Perrin, A. Goldman, S. T. Massie, L. R. Brown, and R. A. Toth, J. Quant. Spectrosc. Radiat. Transfer 48, 469 (1992).

${ }^{19} \mathrm{Q}$. Ma, R. H. Tipping, and C. Boulet (unpublished).

${ }^{20} \mathrm{~J}$. Boissoles, C. Boulet, L. Bonamy, and D. Robert, J. Quant. Spectrosc. Radiat. Transfer 42, 509 (1989). 